OPEN ACCESS

Edited by:

Jean-Pierre Levraud, Institut Pasteur, France

Reviewed by:

Anna Silvia Pistocchi,

University of Milan, Italy

Robert T. Wheeler,

University of Maine, United States

*Correspondence:

Anne-Béatrice Blanc-Potard anne.blanc-potard@univ-montp2.fr

Specialty section:

This article was submitted to Microbes and Innate Immunity,

a section of the journal

Frontiers in Cellular and

Infection Microbiology

Received: 22 July 2021 Accepted: 08 September 2021 Published: 30 September 2021

Citation:

Pont S and Blanc-Potard A-B (2021) Zebrafish Embryo Infection Model to Investigate Pseudomonas aeruginosa Interaction With Innate Immunity and Validate New Therapeutics. Front. Cell. Infect. Microbiol. 11:745851.

doi: 10.3389/fcimb.2021.745851

\section{Zebrafish Embryo Infection Model to Investigate Pseudomonas aeruginosa Interaction With Innate Immunity and Validate New Therapeutics}

\author{
Stéphane Pont ${ }^{1,2}$ and Anne-Béatrice Blanc-Potard ${ }^{1,2 *}$ \\ ${ }^{1}$ Laboratory of Pathogen-Host Interactions (LPHI), Université Montpellier, Montpellier, France, ${ }^{2}$ CNRS, UMR5235, \\ Montpellier, France
}

The opportunistic human pathogen Pseudomonas aeruginosa is responsible for a variety of acute infections and is a major cause of mortality in chronically infected patients with cystic fibrosis (CF). Considering the intrinsic and acquired resistance of $P$. aeruginosa to currently used antibiotics, new therapeutic strategies against this pathogen are urgently needed. Whereas virulence factors of $P$. aeruginosa are well characterized, the interplay between $P$. aeruginosa and the innate immune response during infection remains unclear. Zebrafish embryo is now firmly established as a potent vertebrate model for the study of infectious human diseases, due to strong similarities of its innate immune system with that of humans and the unprecedented possibilities of non-invasive real-time imaging. This model has been successfully developed to investigate the contribution of bacterial and host factors involved in $P$. aeruginosa pathogenesis, as well as rapidly assess the efficacy of anti-Pseudomonas molecules. Importantly, zebrafish embryo appears as the state-ofthe-art model to address in vivo the contribution of innate immunity in the outcome of $P$. aeruginosa infection. Of interest, is the finding that the zebrafish encodes a CFTR channel closely related to human CFTR, which allowed to develop a model to address $P$. aeruginosa pathogenesis, innate immune response, and treatment evaluation in a CF context.

Keywords: Pseudomonas aeruginosa, zebrafish, innate immunity, host-pathogen interactions, drug screening

\section{INTRODUCTION}

Pseudomonas aeruginosa is a mesophilic Gram-negative bacterium able to thrive in very diverse habitats, which is linked to its metabolic versatility, impressive number of regulators, and twocomponent systems (Arai, 2011). P. aeruginosa can colonize a broad range of hosts, from plants to animals including humans (de Bentzmann and Plesiat, 2011), where it is considered as an opportunistic pathogen as it mainly affects compromised patients. This pathogen is a leading cause of nosocomial contaminations capable of causing a myriad of infection types like bacteremia, pneumonia, keratitis, wound, and urinary tract infection, in immunodeficient subjects (e.g., neutropenic, burn, or oncological patients). In addition, most individuals with cystic fibrosis (CF), a genetic disorder caused by mutations of the cystic fibrosis transmembrane conductance 
regulator (CFTR) gene, are colonized by $P$. aeruginosa, which is a major cause of morbidity and mortality in these patients. In this autosomal recessive disease, the function of the chloride channel CFTR is impaired, leading to thick mucus in the lung, providing an environment particularly favorable for $P$. aeruginosa multiplication. CFTR also contributes to the innate immune response, being involved in the bactericidal activity of macrophages (Di et al., 2006; Zhang et al., 2010; Del Porto et al., 2011).

During acute infection, $P$. aeruginosa relies on a wide array of virulence factors, allowing the bacterium to move (flagella), adhere on host cells (pili), degrade host factors (secreted proteases), evade innate immune response, or transmigrate within the organism (Gellatly and Hancock, 2013; Klockgether and Tummler, 2017). Among these weapons, the type three secretion system (T3SS) contributes tremendously to virulence (Hauser, 2009). This nano-syringe allows P. aeruginosa to intoxicate various cell types through the injection of bacterial effectors into host cells. Despite being considered an extracellular pathogen, recent studies have emphasized that $P$. aeruginosa can enter host cells in vivo (Belon et al., 2015; Mittal et al., 2016; Kroken et al., 2018), resulting in a transient phase of intracellular residence, where T3SS also plays a role (Garai et al., 2019). Intracellular residence during infection is an important strategy for bacterial pathogens to hide from the immune system and antibiotics (Lamberti and Surmann, 2021). During long-lasting infections in CF lungs, $P$. aeruginosa is able to shift from a colonizing toward a persistent state, associated with the formation of biofilm, which is extremely hard to eradicate since it protects $P$. aeruginosa against host immune defenses and antibiotics (Faure et al., 2018). Biofilm formation relies on active quorum sensing (QS), a system allowing bacteria to communicate with each other thanks to the release of small signaling molecules.

Pseudomonas aeruginosa is known to be highly resistant to many currently used antibiotics, due to efflux systems expelling intracellular antibiotics, its low outer membrane permeability, and antibiotic-inactivating enzymes (Pang et al., 2019). The recent rising of multidrug-resistant isolates and the major threat they represent at the hospital have led the World Health Organization to classify $P$. aeruginosa as a critical priority for which there is an urgent need for new treatments (Tacconelli et al., 2018). In this line, anti-virulence strategies and phage therapy represent appealing alternatives that are currently being developed to complement antibiotic treatments (Muhlen and Dersch, 2016; Broncano-Lavado et al., 2021).

To investigate $P$. aeruginosa pathogenesis, a plethora of in vivo infection models have been used, ranging from mammals to nonmammalian animals like Caenorhabditis elegans or even plants (Rahme et al., 1997; Lorenz et al., 2016; Kaito et al., 2020). Zebrafish (Danio rerio) is a non-mammalian vertebrate model that is gaining increasing interest in infection studies as it fulfills the advantages of both mammalian and invertebrate models. Zebrafish have a number of advantages, in terms of methodological, financial, and ethical issues over mammalians models, and have been widely used as a model for studying host-pathogen interactions (Torraca and Mostowy, 2018). As a vertebrate, zebrafish is genetically and physically closer to humans than invertebrate models and has an innate immune system closely related to that of humans. In this review, we will focus on the embryo, which is increasingly considered for modeling human infections, including lung diseases, caused by bacterial pathogens (Hernandez et al., 2015; Gomes and Mostowy, 2020). Importantly, the optical transparency of the embryonic stages provides unprecedented opportunities to visualize bacterial infections in real time. Moreover, genetic tools are available to transiently knock down host genes through the generation of morphants using translation-blocking antisense oligonucleotides injected at the one-cell stage or permanently knock down host genes through the generation of mutated fish lines.

During the embryonic stage, only the innate immune system is functional (Masud et al., 2017), making embryos a model of choice to investigate the contribution of innate immune cells upon infection (Torraca et al., 2014). The zebrafish infection model has been used to investigate the involvement of macrophages and neutrophils, which are highly migratory cells, capable of phagocytosis and the subsequent killing of pathogens (Linnerz and Hall, 2020; Rosowski, 2020). Macrophages are the first immune cells to develop in a zebrafish embryo, as early as $22 \mathrm{~h}$ post-fertilization (hpf), and both functional macrophages and neutrophils are present by 30 hpf. Transgenic zebrafish lines with fluorescent macrophages or neutrophils have been generated to visualize specifically the interaction of pathogenic bacteria with these cell lineages. Reporter fish lines can also be generated to monitor the activation of the proinflammatory response in vivo. Moreover, chemical and genetic tools are available to selectively deplete macrophages or neutrophils and investigate their specific role during infection in zebrafish embryos (Rosowski, 2020).

The zebrafish embryo model has been used for the first time to follow $P$. aeruginosa infections and assess its virulence in 2009 by three different groups (Brannon et al., 2009; Clatworthy et al., 2009; Llamas et al., 2009), and detailed methodology for this novel model of infection has been reported (Llamas and van der Sar, 2014). We review here for the first time the various studies implying $P$. aeruginosa carried out in this novel infection model. More specifically, this subject has relevance in addressing $P$. aeruginosa pathogenesis, monitoring interaction with innate immune cells, and evaluating the efficacy of anti-infectious treatments. In addition, the interest of zebrafish embryo as CF model is discussed and future promising studies using this model are underlined.

\section{INFECTION ROUTES FOR PSEUDOMONAS AERUGINOSA IN ZEBRAFISH EMBRYOS}

Infection in zebrafish embryos is most often established through microinjection of bacteria into the embryo, either in the circulation or in closed compartments (Figure 1). The site of microinjection determines whether the infection will rapidly become systemic or will initially remain localized. In addition, 




FIGURE 1 | Different infection routes used with Pseudomonas aeruginosa in the zebrafish embryo. Microinjection offers the possibility to induce either a systemic invasion, by delivering bacteria (in green) directly within the circulation, or a more localized infection, by injecting the pathogen in closed compartments. Immersion of tail-injured embryos in a bacterial bath induces the colonization of the fish tail fin, mimicking a wound infection. Created with BioRender.com.

a bath immersion method has been developed with $P$. aeruginosa (Figure 1).

\section{Microinjection in the Circulation or in Closed Compartments}

In initial experiments, $P$. aeruginosa infections were established by microinjecting bacteria into the bloodstream (injection in caudal vein or duct of Cuvier) of 1 or 2 days post-fertilization (dpf) old embryos, which causes a rapid systemic infection. The detailed handling for injection in the caudal vein has been described (Llamas and van der Sar, 2014).

Microinjection in a closed compartment such as the hindbrain ventricle (HBV), otic vesicle, or tail muscle provides a local infection that facilitates live imaging. Notably, injection in HBV or muscle allowed to visualize in real time macrophages that migrate at the injection site to phagocytose bacteria (Moussouni et al., 2021) (Figure 2). Neutrophil recruitment has also been monitored upon HBV injection (McCarthy et al., 2017). Interestingly, lines of evidence of the development of $P$. aeruginosa microcolonies were reported upon $\mathrm{HBV}$ injection 24 hpi, which have been proposed as precursors of biofilm (Rocker et al., 2015). Accordingly, microcolonies were less frequent and of lower size and volume for a psl mutant, which is defective in the production of Psl exopolysaccharide and thus biofilm formation (Rocker et al., 2015). This study was, however, conducted on fixed and not live embryos. To further evaluate biofilm initiation in vivo, it would be of great interest to follow the formation of microcolonies in real time on live embryos over a period longer than $24 \mathrm{~h}$.

The swim bladder shares similarities with the lung, with an air-epithelial interface that produces epithelial mucus, and localized microinjection in the swim bladder has been used to model mucosal mixed infection with Candida albicans and $P$. aeruginosa (Bergeron et al., 2017). Infection within the swim bladder is usually performed at $4 \mathrm{dpf}$, when the organ starts to inflate and is visible for microinjection.

\section{Bath Immersion}

Microinjection of bacterial cells into zebrafish embryos requires specific expertise and is time consuming. Bath immersion, which is easier to handle, is a natural way of infection for aquatic bacterial pathogens (Rowe et al., 2014), but not for other pathogens. A lethal infection after bath immersion of $3 \mathrm{dpf}$ healthy embryos with concentrated $P$. aeruginosa culture was reported in one report (Diaz-Pascual et al., 2017), but was not reproduced in other studies (Clatworthy et al., 2009; van Soest et al., 2011; Nogaret et al., 2021). Proteomic analysis indicated induction of hypoxia response, but no inflammatory response, in zebrafish embryos exposed to the immersion method, suggesting that healthy larvae suffered from a lack of oxygen when exposed to $P$. aeruginosa by static immersion (Diaz-Pascual et al., 2017). Moreover, the host response was also likely corresponding to a response against bacteria that are outside the fish or in contact with its skin (Diaz-Pascual et al., 2017).

An alternative infection route has been recently proposed with the bath immersion of tail-injured embryos (Poplimont et al., 2020; Nogaret et al., 2021). A rapid (within 24 h) bacterial dose-dependent mortality was observed when 2 dpf embryos were injured at the tail fin before static immersion with $P$. aeruginosa $\mathrm{PAO} 1$ strain. Moreover, a mutant strain known to be attenuated upon microinjection in the caudal vein is similarly attenuated upon bath infection of injured embryos (Nogaret et al., 2021), supporting the reliability of the bath model in assessing $P$. aeruginosa virulence. This infection mode, which could be considered as a model of wound infection, is of interest for drug screening (see below). 




FIGURE 2 | Advantages of zebrafish embryo to assess in vivo the interplay of Pseudomonas aeruginosa with innate immune cells. As shown in the left panel, using specific transgenic lines carrying fluorescently labeled macrophages [e.g., $\operatorname{tg}(m f a p 4: m C h e r r y-F)]$ or neutrophils [e.g., $\operatorname{tg}(m p x: G F P)]$, and thanks to the transparency of the embryo, migration of professional phagocytes toward infectious foci (here HBV) and phagocytosed bacteria can be visualized in real time. The inserted picture is a confocal microscopy image where macrophages express mCherry (red labeling), P. aeruginosa PAO1 expresses GFP (green labeling), and phagocytosed bacteria are indicated by arrows (Moussouni et al., 2021). To assess the respective involvement of macrophages and neutrophils upon $P$. aeruginosa infection, toxin-based (lipochlodronate, nitroreductase system) or genetic tools (morphants) are available to specifically deplete one or the other cell population. In the right panel, images show the efficiency of macrophage depletion by lipochlodronate (lipoCld) in $\operatorname{tg}(m f a p 4: m C h e r r y-F)$ fish line (Moussouni et al., 2021). Survival curves of lipoCld-treated and non-treated embryos infected with PAO1 wild-type stain or PAO1 $\Delta$ oprF mutant are shown (Moussouni et al., 2021). Created with BioRender.com.

\section{INTERPLAY OF PSEUDOMONAS AERUGINOSA WITH THE ZEBRAFISH INNATE IMMUNE SYSTEM}

Infection of zebrafish embryos by $P$. aeruginosa induces an acute infection and lethality of embryos, mostly within $24-30 \mathrm{hpi}$, suggesting that infection is cleared or controlled in surviving embryos. The embryo response to $P$. aeruginosa infection is strongly related to the initial concentration of infection bacteria. The zebrafish is relatively resistant to Pseudomonas, and injection of large inocula (above 1,000 bacteria per embryo) is required to induce host killing. Macrophages and neutrophils can rapidly phagocytose $P$. aeruginosa, suggesting that both phagocytic cell types play a role in protection against infection (Brannon et al., 2009; Clatworthy et al., 2009; Cafora et al., 2019). A $P$. aeruginosa clinical isolate from a CF patient microinjected into the duct of Cuvier was also predominantly found to be associated or engulfed by macrophages within 6 hpi (Kumar et al., 2018). Figure 2 recapitulates some advantages of the zebrafish embryo model to address the role of phagocytic cells during $P$. aeruginosa infection.

Phagocytosis of $P$. aeruginosa, injected in the muscle or HBV, by recruited macrophages has been visualized in real time (Cafora et al., 2019; Moussouni et al., 2021) (Figure 2). Depletion of macrophages, through the use of pu.1 morphants or lipochlodronate, increased the susceptibility of larvae to $P$. aeruginosa (Brannon et al., 2009; Belon et al., 2015; Moussouni et al., 2021) (Figure 2), supporting the role of macrophages in the clearance of $P$. aeruginosa during acute infection. Survival of infected embryos is reduced, in association with increased bacterial burden, in embryos defective for the mitochondrial superoxide dismutase 2 (sod2 morphant), which contributes to generating oxidative stress in phagocytes (Peterman et al., 2015). In addition, the survival of infected embryos is largely reduced in the presence of bafilomycin, which inhibits host vacuolar ATPase, supporting the idea that acid stress within phagocytic cells is important for host defense (Moussouni et al., 2021).

The recruitment of neutrophils at the site of infection has also been observed (Diaz-Pascual et al., 2017; McCarthy et al., 2017). The effect of neutrophil depletion in the outcome of $P$. aeruginosa in zebrafish embryos has not been investigated, but defects in neutrophil migration greatly sensitize embryos to $P$. aeruginosa infections (Rosowski et al., 2016; Houseright et al., 2020). More precisely, embryos defective for Rac2 function, which have neutrophils that are unable to migrate, are highly susceptible to P. aeruginosa (Rosowski et al., 2016). Upon localized otic infection with $P$. aeruginosa, systemic activation and mobilization of neutrophils from hematopoietic tissues is also mediated by $\mathrm{Cxcr} 2$ signaling, a receptor enabling neutrophils to sense the IL8 chemokine (Deng et al., 2013). In addition, the increased susceptibility to $P$. aeruginosa infection observed in zebrafish deficient for $c 3 a .1$, which is homologous to the $\mathrm{C} 3$ component of human complement, is likely due to a neutrophil-intrinsic function of C3, possibly its ability to recruit neutrophils at the infection site (Houseright et al., 2020).

In parallel to the recruitment of phagocytic cells, $P$. aeruginosa triggers a potent proinflammatory response in zebrafish, with an increased expression of cytokines TNF- $\alpha$ 
and IL- $\beta$ that was monitored by RT-qPCR (Clatworthy et al., 2009; Cafora et al., 2019). However, even though a correlation has been made between the intensity of the TNF- $\alpha$ response and the virulence of strains (Clatworthy et al., 2009), it is still unclear whether the mortality of embryos is due to a cytokine storm. An inflammatory response is also supported by global expression profile (see below).

Interestingly, the diguanylate cyclase SadC and the methyltransferase WarA, which interact with the LPS biosynthesis machinery of $P$. aeruginosa to modify the distribution of LPS O antigen, have been involved in neutrophil recruitment (McCarthy et al., 2017). SadC and warA mutants are slightly but significantly attenuated during zebrafish infection at early time post-infection (12 hpi), without being associated with a reduced bacterial load (McCarthy et al., 2017). During infection with sadC or warA mutant in HBV, more neutrophils were recruited to the site of infection, and a higher expression of the LPS-associated proinflammatory cytokine TNF- $\alpha$ was measured as compared to larvae infected with the wild-type strain, suggesting that SadC/WarA modifications of LPS mediate immune evasion in vivo.

Taken together, these studies show the strong contribution of zebrafish's innate immune system in the outcome of $P$. aeruginosa infection and highlight the powerful tools available to decipher the interplay between pathogen and host components. These findings corroborate the role of innate immunity in humans with the increased sensibility of immunocompromised patients, such as neutropenic patients, to $P$. aeruginosa infection (Sadikot et al., 2005).

\section{PSEUDOMONAS AERUGINOSA FACTORS INVOLVED IN PATHOGENESIS IN ZEBRAFISH}

The zebrafish infection model has been used to assess the virulence of various $P$. aeruginosa mutant strains, thus allowing us to evaluate the role of different bacterial factors in this model. The mutants identified as attenuated in this model are summarized in Table 1. Of specific interest, several mutants are as virulent as a wild-type strain in macrophage-depleted embryos, indicating an interplay between these bacterial factors and phagocytes.

\section{T3SS, QS, and Other Classical Virulence Factors}

A PA14 mutant lacking T3SS-structural protein PscD is attenuated upon microinjection into the yolk circulation valley at $2 \mathrm{dpf}$ (Clatworthy et al., 2009). Accordingly, a PAK mutant strain lacking ExsA, which positively regulates T3SS, is also attenuated upon microinjection in the caudal vein of $2 \mathrm{dpf}$ embryos (Brannon et al., 2009). The first effect of T3SS was reported between 4 and 8 hpi, when the PAK strain, but not an exsA mutant, began to proliferate despite an initial phase of bacterial clearance, probably by phagocytes (Brannon et al., 2009).

The virulence of $P$. aeruginosa strains lacking a functional T3SS can be restored upon phagocyte depletion, suggesting that T3SS influences virulence through its effects on phagocytes (Brannon et al., 2009). The dramatic dependence of $P$. aeruginosa on the T3SS to overcome normal phagocyte defenses in the absence of adaptive immunity suggests that the zebrafish may be a useful and relevant model to understand the details of T3SS-phagocyte interactions. The specific involvement of T3SS effectors during infection in the zebrafish model still remains to be investigated.

Two bacterial factors, the inner membrane protein $\mathrm{MgtC}$ and the outer membrane porin OprF, which are involved in the intramacrophage survival of $P$. aeruginosa, have been involved in the regulation of T3SS genes (Garai et al., 2019). Similar to T3SS mutants, $m g t C$ and $o p r F$ mutants (in the PAO1 background) have been shown to be attenuated after microinjection in the caudal vein of $2 \mathrm{dpf}$ embryos in a macrophage-dependent manner (Belon et al., 2015; Moussouni et al., 2021) (Figure 2). Attenuation of oprF mutant has also been reported upon infection by immersion of tail-injured embryos (Nogaret et al., 2021). Interestingly, the infection defect of oprF mutant in zebrafish embryo can be suppressed upon the addition of bafilomycin, an inhibitor of phagosomal acidification, which correlates with a preferential association of $o p r F$ mutant with acidified compartments in cultured macrophages (Moussouni et al., 2021).

LasR and MvfR are transcriptional regulators of QS that control many genes encoding virulence factors. $P$. aeruginosa lasR and $m v f R$ mutants (PA14 background) are attenuated in zebrafish embryos infected at $2 \mathrm{dpf}$ into the yolk circulation valley (Clatworthy et al., 2009).

TABLE 1 | Pseudomonas aeruginosa mutants attenuated in the zebrafish embryo infection model.

\begin{tabular}{|c|c|c|}
\hline Gene name & Site of injection & References \\
\hline exs $A^{\mathrm{a}}, \operatorname{psc} D$ (T3SS) & CV, duct of Cuvier & Brannon et al. (2009); Clatworthy et al. (2009) \\
\hline$o p r F^{\mathrm{a}}$ & CV, HBV, immersion & Moussouni et al. (2021); Nogaret et al. (2021) \\
\hline$m g t C^{a}$ & CV & Belon et al. (2015) \\
\hline$m v f R$, lasR (QS) & Duct of Cuvier & Clatworthy et al. (2009) \\
\hline phz1/2 & Duct of Cuvier & Chand et al. (2011) \\
\hline sarA/warA & HBV & McCarthy et al. (2017) \\
\hline rets, gacs, phoR, cops, bqsS, kinB (TCS) & Duct of Cuvier & Chand et al. (2011) \\
\hline PA2206 (LysR regulator) & CV & Reen et al. (2013) \\
\hline vreR & CV & Llamas et al. (2009) \\
\hline
\end{tabular}

${ }^{a}$ Attenuation in a macrophage-dependent manner. 
Among other putative virulence genes tested, a phz1/2 mutant defective in phenazine-1-carboxylic acid biosynthesis (whose main derivative is pyocyanin) was attenuated for virulence in the zebrafish embryo infection model, suggesting that pyocyanin is a critical virulence factor in this model (Chand et al., 2011), which may interfere with bacterial clearance by phagocytes. In contrast, mutants deficient for elastase production (lasB), flagellar motility $(f l g K)$, alginate $(\operatorname{alg} D)$, or Pel exopolysaccharide (pelA) production were not attenuated during acute infection in zebrafish embryos (Chand et al., 2011).

\section{Two-Component Systems and Transcriptional Regulators}

The role of 60 two-component sensors (PA14 genetic background) has been systematically tested with individual mutants in 50 hpf zebrafish embryos microinjected into the bloodstream through the yolk circulation valley. Six sensors that are required for $P$. aeruginosa virulence were identified (GasS, RetS, PhoR, CopS, BqsS, KinB) (Chand et al., 2011). A deeper analysis of $\operatorname{kin} B$ mutant showed that $\mathrm{KinB}$ is required for acute infection in zebrafish embryos and regulates a number of virulence-associated phenotypes, including QS, pyocyanin production, biofilm formation, and motility (Chand et al., 2011).

Extracytoplasmic function sigma factors are important signalresponsive regulatory proteins in $P$. aeruginosa, and a member of this family, $\sigma^{\text {VreI }}$, promotes the transcription of secretion systems and secreted proteins. The VreR anti-sigma factor, involved in the regulation of $\sigma^{\mathrm{VreI}}$, is required for $P$. aeruginosa virulence in zebrafish embryos, possibly through the modulation of bacterial toxicity toward host cells (Llamas et al., 2009; Otero-Asman et al., 2020).

The non-classical LysR-type transcriptional regulator PA2206 is required for an effective oxidative stress response in $P$. aeruginosa (required for tolerance to $\mathrm{H}_{2} \mathrm{O}_{2}$ in vitro) and was found to be important for the lethality of zebrafish embryos injected at $26 \mathrm{hpf}$ in the blood island (PAO1 derivative) (Reen et al., 2013). Whether the attenuated phenotype of PA2206 mutant in the zebrafish infection model is directly linked to its reduced tolerance to oxidative stress remains to be determined. In addition, the implication of phagocytic cells in the attenuated phenotype has not been investigated.

Cumulatively, the use of diverse mutated strains indicates that the relevance of $P$. aeruginosa factors, such as T3SS and QS, during pathogenesis in zebrafish embryo model is consistent with the results reported in acute models of mice infection (Rumbaugh et al., 1999; Vance et al., 2005; Kumar et al., 2009). In addition, virulence phenotypes in zebrafish corroborate clinical observations in humans, since T3SS has been shown to increase the infection risk and is associated with poor clinical outcomes (Hauser et al., 2002; Ledizet et al., 2012), similar to pyocyanin production upon bloodstream infections (Gupte et al., 2021). On the other hand, similar to what is observed in zebrafish, swimming motility and protease secretion have not been associated with increased pathogenicity and disease severity in humans (Ledizet et al., 2012; Gupte et al., 2021).

\section{EXPRESSION OF HOST AND PSEUDOMONAS AERUGINOSA GENES DURING INFECTION}

The transparency of the zebrafish embryos can be used to monitor bacterial gene expression in vivo using strains with transcriptional fusions with fluorescent reporter genes. Expression in vivo of a $P$. aeruginosa gene regulated by the $\sigma^{\mathrm{VreI}}$ transcriptional factor was observed upon bacterial injection in HBV (Otero-Asman et al., 2020). How activation of $\sigma^{\text {VreI }}$ in response to the host occurs remains to be investigated.

The use of a zebrafish embryo model also allows us to assess the global gene expression of both host and microbe in parallel. This provides a unique opportunity to investigate the molecular mechanisms underlying the interaction between the host's innate immune system and the pathogen.

A global proteomic approach was used to track simultaneously in vivo the pathogen response and host immune response at $22 \mathrm{hpi}$ using $3 \mathrm{dpf}$ zebrafish larvae infected with $P$. aeruginosa PAO1 by immersion (without tail injury) or microinjection (Diaz-Pascual et al., 2017). Some zebrafish metabolic pathways, such as hypoxia response, as well as the integrin signaling pathway and angiogenesis, were exclusively enriched in the larvae exposed by static immersion. In contrast, inflammation mediated by chemokine and cytokine signaling pathways was exclusively enriched in the larvae exposed by injection. Important virulence factors from $P$. aeruginosa, involved in toxin production, T3SS, QS, and the production of extracellular polymeric substances, were enriched only after exposure by injection, which is consistent with the role of these factors during acute infection in this model (Table 1).

A dual host-pathogen transcriptomic analysis was also conducted at a later infection time (3 dpi) on embryos surviving infection using a CF clinical isolate (PASS1) microinjected into the duct of Cuvier at 2 dpf (Kumar et al., 2018). PASS1 displayed increased expression of an array of genes shown previously to be important in pathogenesis, including genes encoding pyoverdine biosynthesis, flagellin, non-hemolytic phospholipase C, proteases, superoxide dismutase, and fimbrial subunits. In addition, phosphate and iron acquisition genes are significantly upregulated in PASS1, suggesting that phosphate and iron are limiting nutrients within the zebrafish host. Regarding the host, proinflammatory genes as chemokine receptors, IL-1 $\beta$, Toll-like receptors, and TNF receptor signaling family were activated. Transcriptional regulators of neutrophil and macrophage phagocytosis were also upregulated, highlighting phagocytosis as a key response mechanism to $P$. aeruginosa infection.

Real-time imaging using zebrafish transgenic lines carrying reporters of inflammatory genes and a cohort of clinical isolates could increase the relevance of these observations, notably by shedding light on the ability of strains from different origins to escape immune detection. In humans, host responses to chronic $P$. aeruginosa infections are indeed complex, ranging from vigorous inflammation ineffective at eradicating infecting bacteria, to relative host tolerance through a dampened activation of host immunity (Faure et al., 2018). 


\section{ROLE OF HOST FACTORS DURING PSEUDOMONAS AERUGINOSA INFECTION: THE CASE OF CFTR}

As indicated above, $P$. aeruginosa infections are a major cause of mortality and morbidity in patients with CF. The CFTR channel has a broad cellular distribution and CF affects multiple organs in humans including the lung, gastrointestinal tract, liver, male reproductive tract, and pancreas.

\section{CFTR in Zebrafish}

The amino acid sequence of zebrafish CFTR (zCFTR) is 55\% identical to human CFTR (hCFTR), and 42 out of 46 sites of mutations found in patients with CF are conserved in zCFTR. The structure of zCFTR was the first CFTR structure to be solved (Zhang and Chen, 2016; Zhang et al., 2017) and is essentially identical to the structure of hCFTR (Liu et al., 2017). Zebrafish $c f t r$ is expressed in the liver, kidney, spleen, and intestine, and $c f t r$ transcripts are detected in cells of the myeloid lineage that includes macrophages and neutrophils (Phennicie et al., 2010).

The role of CFTR in zebrafish was addressed by generating a fish line with frameshift mutation in the $c f t r$ gene (Navis et al., 2013; Liao et al., 2018). In zebrafish, left-right asymmetry requires cilia-driven fluid flow within the lumen of Kupffer's vesicle (KV), and a reduced fluid secretion in cftr mutant impairs KV lumen expansion leading to defects in organ laterality (Navis et al., 2013). Due to male infertility (Liao et al., 2018), the lines have to be maintained at heterozygous stage, and homozygous embryos are screened based on the altered KV morphogenesis (Navis et al., 2013). Interestingly, deregulation of $c f t r$ function in zebrafish causes a phenotype that mirrors other defects present in the human disease such as severe pancreatic dysfunction (Navis and Bagnat, 2015) and hematopoietic defects (Sun et al., 2018), which may correlate with anemia presented by patients with $\mathrm{CF}$.

Zebrafish embryos with cftr-loss-of-function (also called "CF embryos") thus represent a promising model to study the implication of CFTR in innate immune response and mucin secretion. Another way to generate a CF zebrafish model, which has been used in the context of $P$. aeruginosa infection, is through the injection of morpholino that transiently knocks down $c f t r$ gene expression.

\section{Pseudomonas aeruginosa Infection in CF Zebrafish Embryos}

CF zebrafish embryos (cftr morphants) have a 3.5-fold higher number of $P$. aeruginosa PA14 bacterial cells after $8 \mathrm{~h}$ of infection than control embryos (Phennicie et al., 2010). A similar pattern of differences in bacterial burden at early time post-infection was observed with a $P$. aeruginosa CF clinical isolate. However, no effect was reported at later times, and this difference early in the course of infection did not result in higher mortality in the cftr morphants compared with that of the control. On the other hand, in another study carried out with PAO1 strain microinjected in the duct of Cuvier at $48 \mathrm{hpf}$, the mortality of the CF embryos was slightly, but significantly, increased at $20 \mathrm{hpi}$ comparative to control embryos (Cafora et al., 2019). Time laps analyses after HBV injection by confocal microscopy showed several microcolonies at 18 hpi (Rocker et al., 2015), and interestingly, the area of microcolonies formed in CF embryos was reported to be higher than in wild-type fish (Cafora et al., 2019). A deeper analysis of the formation of these microcolonies over a long period would be of interest to determine if they persist, increase in size and harbor biofilm characteristics, or are eliminated.

The production of reactive oxygen species is significantly dampened in cftr morphants compared with control embryos, and a reduction of neutrophil migration toward the injection site is observed in the case of local injection (HBV), supporting a link between CFTR and innate immune response (Phennicie et al., 2010). Moreover, CF embryos present a reduced proinflammatory immune response following bacterial infection in comparison with the wild-type, as shown by significantly reduced TNF- $\alpha$ and IL- $1 \beta$ responses (Phennicie et al., 2010; Cafora et al., 2019).

Taken together, these results indicate that zCFTR contributes, to a moderate extent, to the resistance against $P$. aeruginosa infection. This is likely linked to an alteration of the inflammatory response and could also be related to an alteration of the bactericidal action of innate immune cells (Bernut et al., 2019). Such findings corroborate a reduced clearance of $P$. aeruginosa in the CF mouse model and in patients with $\mathrm{CF}$, which is in part linked to the altered ability of CF macrophages to properly control the inflammatory response and kill bacteria (Hartl et al., 2012). The results in zebrafish rely on a morphant-based CF model and it would be of interest to carry out $P$. aeruginosa infection with a mutated fish line, to strengthen the invalidation of the $c f t r$ gene.

\section{VALIDATION OF ANTI-PSEUDOMONAS STRATEGIES IN ZEBRAFISH}

Aside from being of interest as an infection model, the zebrafish embryo is also suitable for in vivo chemical screening (Zon and Peterson, 2005; Rennekamp and Peterson, 2015), with the advantage that permeability of the larvae allows the entry of small compounds added directly to the fish water. This model, which allows also to address drug toxicity (Eimon and Rubinstein, 2009), has been successfully used for drug testing in the context of infectious diseases (Bernut et al., 2014b). The mode of infection with bath immersion of cut-tailed embryos in 96-well plates is of particular interest for the screening of antiinfectious compounds.

\section{Test of Clinically Used Antibiotics}

Treatment of infected embryos, microinjected at $50 \mathrm{hpf}$ with PA14 in the circulation, with ciprofloxacin $(50 \mu \mathrm{g} / \mathrm{ml})$ or imipenem $(50 \mu \mathrm{g} / \mathrm{ml})$ added in the bath medium, could rescue embryos from lethality (Clatworthy et al., 2009). Protection with ciprofloxacin $(50 \mu \mathrm{g} / \mathrm{ml})$ was also observed with the infection mode of tail-injured embryos (Nogaret et al., 2021). In this case, an antibiotic was added in the bath $2 \mathrm{~h}$ after injury and bacterial 
immersion, at a time when the wound was closed. Interestingly, a significant protective effect, of lower amplitude, was also shown at a much lower dose of $1 \mu \mathrm{g} / \mathrm{ml}$, supporting the pertinence of the model for drug testing.

\section{Validation of Novel Antibacterial Strategies}

Phage therapy using a phage cocktail, found to be efficient to treat $P$. aeruginosa acute infections in mouse and Galleria mellonella larvae, was used in zebrafish embryos (Cafora et al., 2019; Cafora et al., 2020b). Phage therapy, done by injecting a phage cocktail in the yolk sac of PAO1-infected embryos, was shown to reduce lethality, bacterial burden, and the proinflammatory response caused by PAO1 infection at 20 hpi both in wild-type and CF zebrafish (Cafora et al., 2019). In addition, phages alone mitigate inflammation in wild-type and CF zebrafish by reducing the expression levels of proinflammatory cytokines and the neutrophilic recruitment to the infection site (Cafora et al., 2020a).

Anti-virulence strategies have emerged as attractive novel therapeutic approaches that would apply less selective pressure to develop resistance and better preserve microbiota than traditional antimicrobial therapy (Muhlen and Dersch, 2016). In this context, the zebrafish embryo is suitable for testing the efficacy of specific inhibitors of virulence factors that are important for $P$. aeruginosa infection in this model (Table 1). This is the case of QS, which has been proposed as an attractive target to fight $P$. aeruginosa infections using alternative therapies (Smith and Iglewski, 2003). The bath immersion model of injured embryos was used to show the anti-infective potential of a novel anti-QS compound, without exhibiting any toxicity (Nogaret et al., 2021). This compound is an antagonistic analog of $\mathrm{C}_{4}$-HSL (Furiga et al., 2016) that was not previously tested in an animal model. In vitro, $\mathrm{C} 11$ was shown to inhibit $P$. aeruginosa biofilm formation and reduce the expression of QS regulatory/regulated genes (Furiga et al., 2016). Further studies are required to determine how $\mathrm{C} 11$ addition in vivo reduces embryo mortality, which can be due to its ability to downregulate QS gene expression and impair virulence and/or to the fact that initiation of infection at the injured tail shares properties with the initiation of biofilm formation.
Taken together, these studies support the relevance of zebrafish as a first-intention vertebrate model to validate molecules that reduce $P$. aeruginosa pathogenicity and to perform drug screening. In addition, therapeutic molecules can also be tested in a CF context.

\section{CONCLUSION AND PERSPECTIVES}

In the present review, we have recapitulated the multiple points of interest of using zebrafish embryos as an infection model for $P$. aeruginosa (Figure 3). This model appears as a potent firstintention vertebrate model to monitor $P$. aeruginosa pathogenesis and test novel therapeutic strategies. Importantly, this model offers unprecedented opportunities to investigate the role of phagocytic cells and inflammatory response during infection. Whereas the role of alveolar macrophages in internalization and early clearance of $P$. aeruginosa in mouse lung remains controversial (Kooguchi et al., 1998; Cheung et al., 2000), macrophages play a key role during $P$. aeruginosa infection in the zebrafish model, thus allowing to investigate how intraphagocytic stage contributes to dissemination, persistence, and susceptibility to drug treatment.

In addition to these multiple points of interest, zebrafish embryos also offer perspectives to study $P$. aeruginosa clinical isolates and polymicrobial infections (Figure 3). While reference laboratory strains causing acute infections (PAO1, PA14, and PAK) have been widely used in the zebrafish model to gain insights on the host- $P$. aeruginosa interaction in vivo, investigation of the pathogenesis of clinical isolates in this vertebrate model remains very scarce, with only two CF isolates used (Phennicie et al., 2010; Kumar et al., 2018). In the future, a broader and deeper analysis of the behavior of clinical acute or chronic strains would shed light on their pathogenesis and on the pertinence of the model to assess persistence strategies used by CF isolates, as well as investigate in vivo their ability to induce or reduce the production of proinflammatory cytokines.

Zebrafish embryo has been proposed as a novel vertebrate CF model, but the increased sensitivity of $\mathrm{ctr}$ morphants to

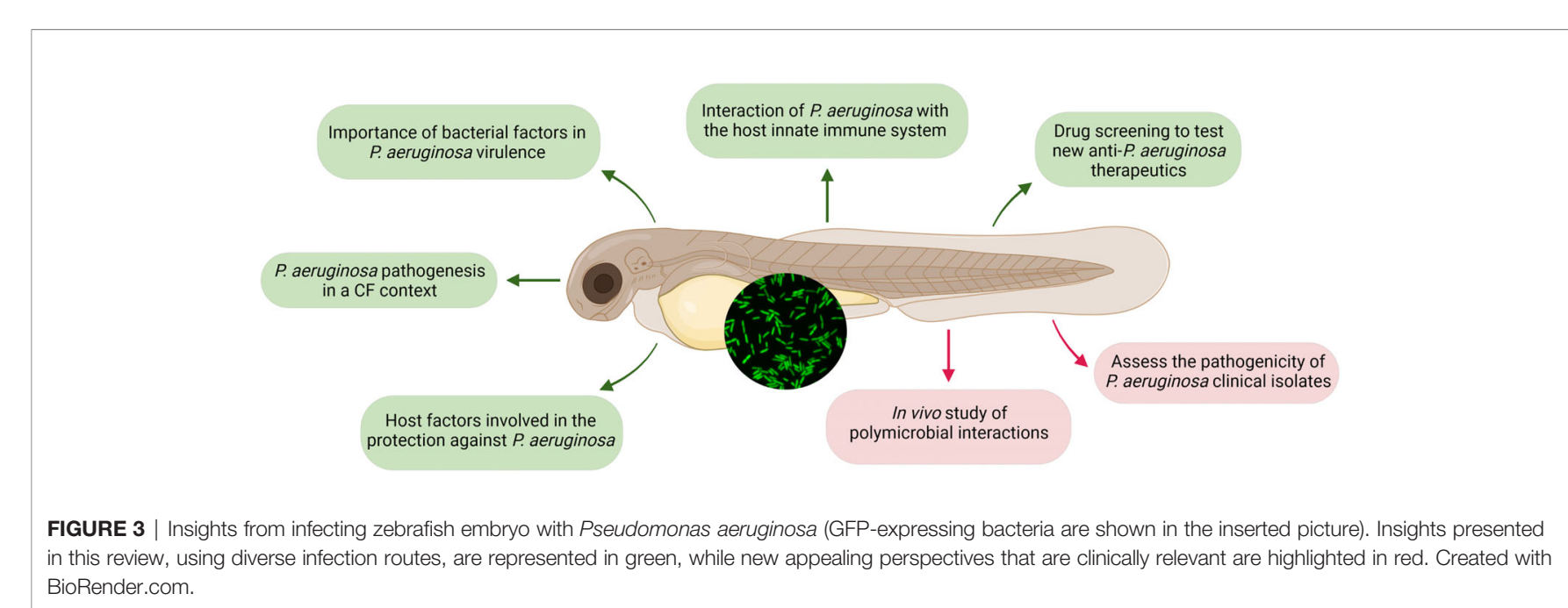


$P$. aeruginosa infection remains moderate, suggesting that the model should be improved, possibly with the use of mutated fish lines. Importantly, zebrafish embryo has been reported as a suitable model for other opportunistic bacterial pathogens found in patients with CF, such as Staphylococcus aureus, Burkholderia cenopacia, and Mycobacterium abscessus (Prajsnar et al., 2008; Vergunst et al., 2010; Bernut et al., 2014a). The zebrafish embryo offers interesting opportunities for future studies of $P$. aeruginosa in the context of microbial communities, as well as in the immune response against polymicrobial infection (Bergeron et al., 2017). It is well known that multiple microbial species can interact together within a given microenvironment, particularly within mixed biofilms, and that it can contribute to increased disease severity during polymicrobial infections (Briaud et al., 2019). Co-infection of C. albicans and $P$. aeruginosa in zebrafish swim bladder, proposed as a suitable site to model mucosal lung, showed increased proliferation of both pathogens, as well as potentiated hyphal penetration through epithelial barriers and increased the host inflammatory response (Bergeron et al., 2017). This suggests in vivo cross talk that benefits both organisms to the detriment of the host and sheds light on the effect of cross-kingdom interactions on infection outcome. In

\section{REFERENCES}

Arai, H. (2011). Regulation and Function of Versatile Aerobic and Anaerobic Respiratory Metabolism in Pseudomonas Aeruginosa. Front. Microbiol. 2, 103. doi: $10.3389 /$ fmicb.2011.00103

Belon, C., Soscia, C., Bernut, A., Laubier, A., Bleves, S., and Blanc-Potard, A. B. (2015). A Macrophage Subversion Factor Is Shared by Intracellular and Extracellular Pathogens. PloS Pathog. 11, e1004969. doi: 10.1371/ journal.ppat.1004969

Bergeron, A. C., Seman, B. G., Hammond, J. H., Archambault, L. S., Hogan, D. A., and Wheeler, R. T. (2017). Candida Albicans and Pseudomonas Aeruginosa Interact To Enhance Virulence of Mucosal Infection in Transparent Zebrafish. Infect. Immun. 85, e00475. doi: 10.1128/IAI.00475-17

Bernut, A., Dupont, C., Ogryzko, N. V., Neyret, A., Herrmann, J. L., Floto, R. A., et al. (2019). CFTR Protects Against Mycobacterium Abscessus Infection by Fine-Tuning Host Oxidative Defenses. Cell Rep. 26, 1828-182+. doi: 10.1016/ j.celrep.2019.01.071

Bernut, A., Herrmann, J. L., Kissa, K., Dubremetz, J. F., Gaillard, J. L., Lutfalla, G., et al. (2014a). Mycobacterium Abscessus Cording Prevents Phagocytosis and Promotes Abscess Formation. Proc. Natl. Acad. Sci. United States America. 111, E943-E952. doi: 10.1073/pnas.1321390111

Bernut, A., Le Moigne, V., Lesne, T., Lutfalla, G., Herrmann, J. L., and Kremer, L. (2014b). In Vivo Assessment of Drug Efficacy Against Mycobacterium Abscessus Using the Embryonic Zebrafish Test System. Antimicrob Agents Chemother. 58, 4054-4063. doi: 10.1128/AAC.00142-14

Brannon, M. K., Davis, J. M., Mathias, J. R., Hall, C. J., Emerson, J. C., Crosier, P. S., et al. (2009). Pseudomonas Aeruginosa Type III Secretion System Interacts With Phagocytes to Modulate Systemic Infection of Zebrafish Embryos. Cell. Microbiol. 11, 755-768. doi: 10.1111/j.1462-5822.2009.01288.x

Briaud, P., Camus, L., Bastien, S., Doleans-Jordheim, A., Vandenesch, F., and Moreau, K. (2019). Coexistence With Pseudomonas Aeruginosa Alters Staphylococcus Aureus Transcriptome, Antibiotic Resistance and Internalization Into Epithelial Cells. Sci. Rep. 9, 16564. doi: 10.1038/s41598019-52975-z

Broncano-Lavado, A., Santamaria-Corral, G., Esteban, J., and Garcia-Quintanilla, M. (2021). Advances in Bacteriophage Therapy Against Relevant MultiDrugResistant Pathogens. Antibiot (Basel). 10, 672. doi: 10.3390/ antibiotics10060672

Cafora, M., Brix, A., Forti, F., Loberto, N., Aureli, M., Briani, F., et al (2020a). Phages as Immunomodulators and Their Promising Use as Anti-Inflammatory addition, the use of gnotobiotic zebrafish colonized with $P$. aeruginosa also demonstrated that zebrafish provides opportunities to explore how habitat influences the establishment of microbiota, and how microbial dynamics in vivo affect host biology (Rawls et al., 2007).

\section{AUTHOR CONTRIBUTIONS}

SP and A-BB-P wrote the manuscript. SP drew the figures. All authors contributed to the article and approved the submitted version.

\section{ACKNOWLEDGMENTS}

Our work is supported by Vaincre La Mucoviscidose and Association Gregory Lemarchal (RF20190502411, RF20200502703). SP is supported by Vaincre La Mucoviscidose and Association Gregory Lemarchal (RF20200502703). We thank Pauline Nogaret (Montpellier) for feedback on the manuscript and on the figures.

Agents in a Cftr Loss-of-Function Zebrafish Model. J. Cystic Fibrosis. S15691993, 30927. doi: 10.1016/j.jcf.2020.11.017

Cafora, M., Deflorian, G., Forti, F., Ferrari, L., Binelli, G., Briani, F., et al. (2019). Phage Therapy Against Pseudomonas Aeruginosa Infections in a Cystic Fibrosis Zebrafish Model. Sci. Rep. 9, 1527. doi: 10.1038/s41598-018-37636-x

Cafora, M., Forti, F., Briani, F., Ghisotti, D., and Pistocchi, A. (2020b). Phage Therapy Application to Counteract Pseudomonas Aeruginosa Infection in Cystic Fibrosis Zebrafish Embryos. J. Visualized Exp JoVE. 159. doi: 10.3791/ 61275

Chand, N. S., Lee, J. S., Clatworthy, A. E., Golas, A. J., Smith, R. S., and Hung, D. T. (2011). The Sensor Kinase KinB Regulates Virulence in Acute Pseudomonas Aeruginosa Infection. J. Bacteriol. 193, 2989-2999. doi: 10.1128/JB.01546-10

Cheung, D. O., Halsey, K., and Speert, D. P. (2000). Role of Pulmonary Alveolar Macrophages in Defense of the Lung Against Pseudomonas Aeruginosa. Infect. Immun. 68, 4585-4592. doi: 10.1128/IAI.68.8.4585-4592.2000

Clatworthy, A. E., Lee, J. S., Leibman, M., Kostun, Z., Davidson, A. J., and Hung, D. T. (2009). Pseudomonas Aeruginosa Infection of Zebrafish Involves Both Host and Pathogen Determinants. Infect. Immun. 77, 1293-1303. doi: 10.1128/ IAI.01181-08

de Bentzmann, S., and Plesiat, P. (2011). The Pseudomonas Aeruginosa Opportunistic Pathogen and Human Infections. Environ. Microbiol. 13, 1655-1665. doi: 10.1111/j.1462-2920.2011.02469.x

Del Porto, P., Cifani, N., Guarnieri, S., Di Domenico, E. G., Mariggio, M. A., Spadaro, F., et al. (2011). Dysfunctional CFTR Alters the Bactericidal Activity of Human Macrophages Against Pseudomonas Aeruginosa. PloS One 6, e19970. doi: 10.1371/journal.pone.0019970

Deng, Q., Sarris, M., Bennin, D. A., Green, J. M., Herbomel, P., and Huttenlocher, A. (2013). Localized Bacterial Infection Induces Systemic Activation of Neutrophils Through Cxcr2 Signaling in Zebrafish. J. Leukocyte Biol. 93, 761-769. doi: 10.1189/jlb.1012534

Diaz-Pascual, F., Ortiz-Severin, J., Varas, M. A., Allende, M. L., and Chavez, F. P. (2017). In Vivo Host-Pathogen Interaction as Revealed by Global Proteomic Profiling of Zebrafish Larvae. Front. Cell. Infect. Microbiol. 7. doi: 10.3389/ fcimb.2017.00334

Di, A., Brown, M. E., Deriy, L. V., Li, C., Szeto, F. L., Chen, Y., et al. (2006). CFTR Regulates Phagosome Acidification in Macrophages and Alters Bactericidal Activity. Nat. Cell Biol. 8, 933-944. doi: 10.1038/ncb1456

Eimon, P. M., and Rubinstein, A. L. (2009). The Use of In Vivo Zebrafish Assays in Drug Toxicity Screening. Expert Opin. Drug Met. 5, 393-401. doi: 10.1517/ 17425250902882128 
Faure, E., Kwong, K., and Nguyen, D. (2018). Pseudomonas Aeruginosa in Chronic Lung Infections: How to Adapt Within the Host? Front. Immunol. 9. doi: $10.3389 /$ fimmu.2018.02416

Furiga, A., Lajoie, B., El Hage, S., Baziard, G., and Roques, C. (2016). Impairment of Pseudomonas Aeruginosa Biofilm Resistance to Antibiotics by Combining the Drugs With a New Quorum-Sensing Inhibitor. Antimicrob Agents Chemother. 60, 1676-1686. doi: 10.1128/AAC.02533-15

Garai, P., Berry, L., Moussouni, M., Bleves, S., and Blanc-Potard, A. B. (2019). Killing From the Inside: Intracellular Role of T3SS in the Fate of Pseudomonas Aeruginosa Within Macrophages Revealed by mgtC and oprF Mutants. PloS Pathog. 15, e1007812. doi: 10.1371/journal.ppat.1007812

Gellatly, S. L., and Hancock, R. E. W. (2013). Pseudomonas Aeruginosa: New Insights Into Pathogenesis and Host Defenses. Pathog. Dis. 67, 159-173. doi: 10.1111/2049-632X.12033

Gomes, M. C., and Mostowy, S. (2020). The Case for Modeling Human Infection in Zebrafish. Trends Microbiol. 28, 10-18. doi: 10.1016/j.tim.2019.08.005

Gupte, A., Jyot, J., Ravi, M., and Ramphal, R. (2021). High Pyocyanin Production and non-Motility of Pseudomonas Aeruginosa Isolates are Correlated With Septic Shock or Death in Bacteremic Patients. PloS One 16, e0253259. doi: 10.1371/journal.pone.0253259

Hartl, D., Gaggar, A., Bruscia, E., Hector, A., Marcos, V., Jung, A., et al. (2012). Innate Immunity in Cystic Fibrosis Lung Disease. J. Cystic Fibrosis Off. J. Eur. Cystic Fibrosis Soc. 11, 363-382. doi: 10.1016/j.jcf.2012.07.003

Hauser, A. R. (2009). The Type III Secretion System of Pseudomonas Aeruginosa: Infection by Injection. Nat. Rev. Microbiol. 7, 654-665. doi: 10.1038/ nrmicro2199

Hauser, A. R., Cobb, E., Bodi, M., Mariscal, D., Valles, J., Engel, J. N., et al. (2002). Type III Protein Secretion is Associated With Poor Clinical Outcomes in Patients With Ventilator-Associated Pneumonia Caused by Pseudomonas Aeruginosa. Crit. Care Med. 30, 521-528. doi: 10.1097/00003246-20020300000005

Hernandez, Y. L., Yero, D., Pinos-Rodriguez, J. M., and Gibert, I. (2015). Animals Devoid of Pulmonary System as Infection Models in the Study of Lung Bacterial Pathogens. Front. Microbiol. 6. doi: 10.3389/fmicb.2015.00038

Houseright, R. A., Rosowski, E. E., Lam, P. Y., Tauzin, S. J. M., Mulvaney, O., Dewey, C. N., et al. (2020). Cell Type Specific Gene Expression Profiling Reveals a Role for Complement Component C3 in Neutrophil Responses to Tissue Damage. Sci. Rep. 10, 15716. doi: 10.1038/s41598-020-72750-9

Kaito, C., Murakami, K., Imai, L., and Furuta, K. (2020). Animal Infection Models Using non-Mammals. Microbiol. Immunol. 64, 585-592. doi: 10.1111/13480421.12834

Klockgether, J., and Tummler, B. (2017). Recent Advances in Understanding Pseudomonas Aeruginosa as a Pathogen. F1000Res. 6, 1261. doi: 10.12688/ f1000research.10506.1

Kooguchi, K., Hashimoto, S., Kobayashi, A., Kitamura, Y., Kudoh, I., WienerKronish, J., et al. (1998). Role of Alveolar Macrophages in Initiation and Regulation of Inflammation in Pseudomonas Aeruginosa Pneumonia. Infect. Immun. 66, 3164-3169. doi: 10.1128/IAI.66.7.3164-3169.1998

Kroken, A. R., Chen, C. K., Evans, D. J., Yahr, T. L., and Fleiszig, S. M. J. (2018). The Impact of ExoS on Pseudomonas Aeruginosa Internalization by Epithelial Cells Is Independent of fleQ and Correlates With Bistability of Type Three Secretion System Gene Expression. mBio. 9, 00668. doi: 10.1128/mBio.0066818

Kumar, R., Chhibber, S., and Harjai, K. (2009). Quorum Sensing is Necessary for the Virulence of Pseudomonas Aeruginosa During Urinary Tract Infection. Kidney Int. 76, 286-292. doi: 10.1038/ki.2009.183

Kumar, S. S., Tandberg, J. I., Penesyan, A., Elbourne, L. D. H., Suarez-Bosche, N., Don, E., et al. (2018). Dual Transcriptomics of Host-Pathogen Interaction of Cystic Fibrosis Isolate Pseudomonas Aeruginosa PASS1 With Zebrafish. Front. Cell. Infect. Microbiol. 8, 406. doi: 10.3389/fcimb.2018.00406

Lamberti, Y., and Surmann, K. (2021). The Intracellular Phase of Extracellular Respiratory Tract Bacterial Pathogens and its Role on Pathogen-Host Interactions During Infection. Curr. Opin. Infect. Dis. 34, 197-205. doi: 10.1097/QCO.0000000000000727

Ledizet, M., Murray, T. S., Puttagunta, S., Slade, M. D., Quagliarello, V. J., and Kazmierczak, B. I. (2012). The Ability of Virulence Factor Expression by Pseudomonas Aeruginosa to Predict Clinical Disease in Hospitalized Patients. PloS One 7, e49578. doi: 10.1371/journal.pone.0049578
Liao, H., Chen, Y., Li, Y., Xue, S., Liu, M., Lin, Z., et al. (2018). CFTR is Required for the Migration of Primordial Germ Cells During Zebrafish Early Embryogenesis. Reprod. 156, 261-268. doi: 10.1530/REP-17-0681

Linnerz, T., and Hall, C. J. (2020). The Diverse Roles of Phagocytes During Bacterial and Fungal Infections and Sterile Inflammation: Lessons From Zebrafish. Front. Immunol. 11, 1094. doi: 10.3389/fimmu.2020.01094

Liu, F., Zhang, Z., Csanady, L., Gadsby, D. C., and Chen, J. (2017). Molecular Structure of the Human CFTR Ion Channel. Cell. 169, 85-95 e88. doi: 10.1016/ j.cell.2017.02.024

Llamas, M. A., and van der Sar, A. M. (2014). Assessing Pseudomonas Virulence With Nonmammalian Host: Zebrafish. Methods Mol. Biol. 1149, 709-721. doi: 10.1007/978-1-4939-0473-0_55

Llamas, M. A., van der Sar, A., Chu, B. C., Sparrius, M., Vogel, H. J., and Bitter, W. (2009). A Novel Extracytoplasmic Function (ECF) Sigma Factor Regulates Virulence in Pseudomonas Aeruginosa. PloS Pathog. 5, e1000572. doi: 10.1371/ journal.ppat. 1000572

Lorenz, A., Pawar, V., Haussler, S., and Weiss, S. (2016). Insights Into HostPathogen Interactions From State-of-the-Art Animal Models of Respiratory Pseudomonas Aeruginosa Infections. FEBS Lett. 590, 3941-3959. doi: 10.1002/ 1873-3468.12454

Masud, S., Torraca, V., and Meijer, A. H. (2017). Modeling Infectious Diseases in the Context of a Developing Immune System. Curr. Topics Dev. Biol. 124, $277-$ 329. doi: 10.1016/bs.ctdb.2016.10.006

McCarthy, R. R., Mazon-Moya, M. J., Moscoso, J. A., Hao, Y., Lam, J. S., Bordi, C., et al. (2017). Cyclic-Di-GMP Regulates Lipopolysaccharide Modification and Contributes to Pseudomonas Aeruginosa Immune Evasion. Nat. Microbiol. 2, 17027. doi: $10.1038 /$ nmicrobiol.2017.27

Mittal, R., Lisi, C. V., Kumari, H., Grati, M., Blackwelder, P., Yan, D., et al. (2016). Otopathogenic Pseudomonas Aeruginosa Enters and Survives Inside Macrophages. Front. Microbiol. 7, 1828. doi: 10.3389/fmicb.2016.01828

Moussouni, M., Berry, L., Sipka, T., Nguyen-Chi, M., and Blanc-Potard, A. B. (2021). Pseudomonas Aeruginosa OprF Plays a Role in Resistance to Macrophage Clearance During Acute Infection. Sci. Rep. 11, 359. doi: 10.1038/s41598-020-79678-0

Muhlen, S., and Dersch, P. (2016). Anti-Virulence Strategies to Target Bacterial Infections. Curr. Top. Microbiol. Immunol. 398, 147-183. doi: 10.1007/ 82_2015_490

Navis, A., and Bagnat, M. (2015). Loss of Cftr Function Leads to Pancreatic Destruction in Larval Zebrafish. Dev. Biol. 399, 237-248. doi: 10.1016/ j.ydbio.2014.12.034

Navis, A., Marjoram, L., and Bagnat, M. (2013). Cftr Controls Lumen Expansion and Function of Kupffer's Vesicle in Zebrafish. Dev. 140, 1703-1712. doi: 10.1242/dev.091819

Nogaret, P., El Garah, F., and Blanc-Potard, A. B. (2021). A Novel Infection Protocol in Zebrafish Embryo to Assess Pseudomonas Aeruginosa Virulence and Validate Efficacy of a Quorum Sensing Inhibitor In Vivo. Pathog. 10, 401. doi: 10.3390/pathogens10040401

Otero-Asman, J. R., Quesada, J. M., Jim, K. K., Ocampo-Sosa, A., Civantos, C., Bitter, W., et al. (2020). The Extracytoplasmic Function Sigma Factor Sigma (VreI) is Active During Infection and Contributes to Phosphate StarvationInduced Virulence of Pseudomonas Aeruginosa. Sci. Rep. 10, 3139. doi: 10.1038/s41598-020-60197-x

Pang, Z., Raudonis, R., Glick, B. R., Lin, T. J., and Cheng, Z. Y. (2019). Antibiotic Resistance in Pseudomonas Aeruginosa: Mechanisms and Alternative Therapeutic Strategies. Biotechnol. Adv. 37, 177-192. doi: 10.1016/ j.biotechadv.2018.11.013

Peterman, E. M., Sullivan, C., Goody, M. F., Rodriguez-Nunez, I., Yoder, J. A., and Kim, C. H. (2015). Neutralization of Mitochondrial Superoxide by Superoxide Dismutase 2 Promotes Bacterial Clearance and Regulates Phagocyte Numbers in Zebrafish. Infect. Immun. 83, 430-440. doi: 10.1128/IAI.02245-14

Phennicie, R. T., Sullivan, M. J., Singer, J. T., Yoder, J. A., and Kim, C. H. (2010). Specific Resistance to Pseudomonas Aeruginosa Infection in Zebrafish is Mediated by the Cystic Fibrosis Transmembrane Conductance Regulator. Infect. Immun. 78, 4542-4550. doi: 10.1128/IAI.00302-10

Poplimont, H., Georgantzoglou, A., Boulch, M., Walker, H. A., Coombs, C., Papaleonidopoulou, F., et al. (2020). Neutrophil Swarming in Damaged Tissue Is Orchestrated by Connexins and Cooperative Calcium Alarm Signals. Curr. Biol. Cb. 30, 2761-2776 e2767. doi: 10.1016/j.cub.2020.05.030 
Prajsnar, T. K., Cunliffe, V. T., Foster, S. J., and Renshaw, S. A. (2008). A Novel Vertebrate Model of Staphylococcus Aureus Infection Reveals PhagocyteDependent Resistance of Zebrafish to non-Host Specialized Pathogens. Cell. Microbiol. 10, 2312-2325. doi: 10.1111/j.1462-5822.2008.01213.x

Rahme, L. G., Tan, M. W., Le, L., Wong, S. M., Tompkins, R. G., Calderwood, S. B., et al. (1997). Use of Model Plant Hosts to Identify Pseudomonas Aeruginosa Virulence Factors. Proc. Natl. Acad. Sci. United States America. 94, 1324513250. doi: 10.1073/pnas.94.24.13245

Rawls, J. F., Mahowald, M. A., Goodman, A. L., Trent, C. M., and Gordon, J. I. (2007). In Vivo Imaging and Genetic Analysis Link Bacterial Motility and Symbiosis in the Zebrafish Gut. Proc. Natl. Acad. Sci. United States America. 104, 7622-7627. doi: 10.1073/pnas.0702386104

Reen, F. J., Haynes, J. M., Mooij, M. J., and O'Gara, F. (2013). A non-Classical LysR-Type Transcriptional Regulator PA2206 is Required for an Effective Oxidative Stress Response in Pseudomonas Aeruginosa. PloS One 8, e54479. doi: 10.1371/journal.pone.0054479

Rennekamp, A. J., and Peterson, R. T. (2015). 15 Years of Zebrafish Chemical Screening. Curr. Opin. In Chem. Biol. 24, 58-70. doi: 10.1016/j.cbpa.2014.10.025

Rocker, A. J., Weiss, A. R., Lam, J. S., Van Raay, T. J., and Khursigara, C. M. (2015). Visualizing and Quantifying Pseudomonas Aeruginosa Infection in the Hindbrain Ventricle of Zebrafish Using Confocal Laser Scanning Microscopy. J. Microbiol. Methods 117, 85-94. doi: 10.1016/j.mimet.2015.07.013

Rosowski, E. E. (2020). Illuminating Macrophage Contributions to Host-Pathogen Interactions In Vivo: The Power of Zebrafish. Infect. And Immun. 88, e00906. doi: 10.1128/IAI.00906-19

Rosowski, E. E., Deng, Q., Keller, N. P., and Huttenlocher, A. (2016). Rac2 Functions in Both Neutrophils and Macrophages To Mediate Motility and Host Defense in Larval Zebrafish. J. Immunol. 197, 4780-4790. doi: 10.4049/ jimmunol.1600928

Rowe, H. M., Withey, J. H., and Neely, M. N. (2014). Zebrafish as a Model for Zoonotic Aquatic Pathogens. Dev. And Comp. Immunol. 46, 96-107. doi: 10.1016/j.dci.2014.02.014

Rumbaugh, K. P., Griswold, J. A., and Hamood, A. N. (1999). Contribution of the Regulatory Gene lasR to the Pathogenesis of Pseudomonas Aeruginosa Infection of Burned Mice. J. Of Burn Care Rehabil. 20, 42-49. doi: 10.1097/ 00004630-199901001-00008

Sadikot, R. T., Blackwell, T. S., Christman, J. W., and Prince, A. S. (2005). Pathogen-Host Interactions in Pseudomonas Aeruginosa Pneumonia. Am. J. Of Respir. And Crit. Care Med. 171, 1209-1223. doi: 10.1164/rccm.2004081044 SO

Smith, R. S., and Iglewski, B. H. (2003). Pseudomonas Aeruginosa Quorum Sensing as a Potential Antimicrobial Target. J. Clin. Invest. 112, 1460-1465. doi: 10.1172/JCI200320364

Sun, H., Wang, Y., Zhang, J., Chen, Y., Liu, Y., Lin, Z., et al. (2018). CFTR Mutation Enhances Dishevelled Degradation and Results in Impairment of Wnt-Dependent Hematopoiesis. Cell Death Dis. 9, 275. doi: 10.1038/s41419018-0311-9

Tacconelli, E., Carrara, E., Savoldi, A., Harbarth, S., Mendelson, M., Monnet, D. L., et al. (2018). Discovery, Research, and Development of New Antibiotics: The
WHO Priority List of Antibiotic-Resistant Bacteria and Tuberculosis. Lancet Infect. Dis. 18, 318-327. doi: 10.1016/S1473-3099(17)30753-3

Torraca, V., Masud, S., Spaink, H. P., and Meijer, A. H. (2014). MacrophagePathogen Interactions in Infectious Diseases: New Therapeutic Insights From the Zebrafish Host Model. Dis. Models Mech. 7, 785-797. doi: 10.1242/ dmm.015594

Torraca, V., and Mostowy, S. (2018). Zebrafish Infection: From Pathogenesis to Cell Biology. Trends Cell Biol. 28, 143-156. doi: 10.1016/j.tcb.2017.10.002

Vance, R. E., Rietsch, A., and Mekalanos, J. J. (2005). Role of the Type III Secreted Exoenzymes S, T, and Y in Systemic Spread of Pseudomonas Aeruginosa PAO1 In Vivo. Infect. Immun. 73, 1706-1713. doi: 10.1128/IAI.73.3.17061713.2005

van Soest, J. J., Stockhammer, O. W., Ordas, A., Bloemberg, G. V., Spaink, H. P., and Meijer, A. H. (2011). Comparison of Static Immersion and Intravenous Injection Systems for Exposure of Zebrafish Embryos to the Natural Pathogen Edwardsiella Tarda. BMC Immunol. 12, 58. doi: 10.1186/1471-2172-12-58

Vergunst, A. C., Meijer, A. H., Renshaw, S. A., and O'Callaghan, D. (2010). Burkholderia Cenocepacia Creates an Intramacrophage Replication Niche in Zebrafish Embryos, Followed by Bacterial Dissemination and Establishment of Systemic Infection. Infect. Immun. 78, 1495-1508. doi: 10.1128/IAI.00743-09

Zhang, Z., and Chen, J. (2016). Atomic Structure of the Cystic Fibrosis Transmembrane Conductance Regulator. Cell. 167, 1586-1597 e1589. doi: 10.1016/j.cell.2016.11.014

Zhang, Y., Li, X., Grassme, H., Doring, G., and Gulbins, E. (2010). Alterations in Ceramide Concentration and $\mathrm{pH}$ Determine the Release of Reactive Oxygen Species by Cftr-Deficient Macrophages on Infection. J. Immunol. 184, 51045111. doi: 10.4049/jimmunol.0902851

Zhang, Z., Liu, F., and Chen, J. (2017). Conformational Changes of CFTR Upon Phosphorylation and ATP Binding. Cell. 170, 483-491 e488. doi: 10.1016/ j.cell.2017.06.041

Zon, L. I., and Peterson, R. T. (2005). In Vivo Drug Discovery in the Zebrafish. Nat. Rev. Drug discovery. 4, 35-44. doi: 10.1038/nrd1606

Conflict of Interest: The authors declare that the research was conducted in the absence of any commercial or financial relationships that could be construed as a potential conflict of interest.

Publisher's Note: All claims expressed in this article are solely those of the authors and do not necessarily represent those of their affiliated organizations, or those of the publisher, the editors and the reviewers. Any product that may be evaluated in this article, or claim that may be made by its manufacturer, is not guaranteed or endorsed by the publisher.

Copyright (c) 2021 Pont and Blanc-Potard. This is an open-access article distributed under the terms of the Creative Commons Attribution License (CC BY). The use, distribution or reproduction in other forums is permitted, provided the original author(s) and the copyright owner(s) are credited and that the original publication in this journal is cited, in accordance with accepted academic practice. No use, distribution or reproduction is permitted which does not comply with these terms. 